\title{
WAVES, CORIOLIS FORCE AND THE DYNAMO EFFECT
}

\author{
Swadesh M. Mahajan \\ Institute for Fusion Studies, The University of Texas, Austin, Texas 78712, USA. \\ and \\ Pablo D. Mininni ${ }^{1}$ \\ Advanced Study Program, National Center for Atmospheric Research, P.O.Box 3000, Boulder CO 80307, USA. \\ and \\ Daniel O. Gómez ${ }^{2}$ \\ Departamento de Física, Facultad de Ciencias Exactas y Naturales, Universidad de Buenos Aires, \\ Ciudad Universitaria, 1428 Buenos Aires, Argentina. \\ dgomez@df.uba.ar
}

\begin{abstract}
Dynamo activity caused by waves in a rotating magneto-plasma is investigated. In astrophysical environments such as accretion disks and at sufficiently small spatial scales, the Hall effect is likely to play an important role. It is shown that a combination of the Coriolis force and Hall effect can produce a finite $\alpha$-effect by generating net helicity in the small scales. The shear/ion-cyclotron normal mode of the Hall plasma is the dominant contributor to the dynamo action for short scale motions.
\end{abstract}

Subject headings: MHD — magnetic fields — stars: magnetic fields — galaxies: magnetic fields

\section{INTRODUCTION}

In astrophysical objects, large scale magnetic fields are thought to be generated by helical turbulence (the so-called $\alpha$-effect) and differential rotation (the $\Omega$ effect) (see Meneguzzi, Frisch, \& Pouquet (1981); Brandenburg (2001)). However, note that a large scale magnetic field can in some cases be generated without fully helical turbulence or a net $\alpha$-effect. Within the mean field approximation, there are physical effects which contribute to the mean electromotive force even when the $\alpha$ coefficient is zero. A shear turbulent flow (Urpin 2002), the $\omega \times j$ term (Rädler 1969; Geppert \& Rheinhardt 2002), or magnetic instabilities in a

\footnotetext{
1 also at Departamento de Física, Facultad de Ciencias Exactas y Naturales, Universidad de Buenos Aires, Ciudad Universitaria, 1428 Buenos Aires, Argentina.

${ }^{2}$ also at Instituto de Astronomía y Física del Espacio, Ciudad Universitaria, 1428 Buenos Aires, Argentina.
}

stably stratified atmosphere (Spruit 2002), are some of the examples reported in the literature.

Helicity is naturally imparted to a rotating fluid by the Coriolis force (Moffatt 1970a,b, 1972, 1978). However, at sufficiently small scales, where the Rossby number

$$
R_{S}=\frac{U_{0}}{2 L_{0} \Omega}
$$

is larger than unity ( $U_{0}$ and $L_{0}$ are characteristic velocities and lengths, and $\Omega$ is the rotation rate), the Coriolis force and the resulting induced helicity might become negligibly small. Nonetheless, it is worth noting that in helical turbulent flows, the kinetic helicity develops a direct cascade along with the energy (Cheng et al. 2003; Gómez \& Mininni 2004). Therefore, some small scale turbulent flows can still be helical, even though the source of helicity remains at much larger spatial scales. 
The Hall effect introduces a definite handedness or helicity on small scale fluid motions (Wardle (1999), Balbus \& Terquem (2001)), since the mirror symmetry in the induction equation is broken (see Eqn (3) below). Therefore, one should expect a net $\alpha$-effect in a Hall plasma. The Hall effect becomes relevant whenever the Hall length scale

$$
\lambda=\frac{c}{\omega_{p i}} \frac{U_{A}}{U_{0}} .
$$

is larger than the dissipation scale, a category to which several objects of astrophysical interest belong (Mininni, Gómez, \& Mahajan 2002a, 2003b). Here, $U_{A}$ is the characteristic Alfvénic speed, $c$ is the speed of light, and $\omega_{p i}$ is the ion plasma frequency.

In $\oint 2$ we write down the Hall MHD equations. The normal modes sustained in this system are derived and listed in $\S 3$. In $\S 4$ we briefly summarize the role of normal mode fluctuations on MHD dynamos, and in $\S 5$ this concept is extended to Hall MHD. In $\S 6$ we show the effect of rotation on the $\alpha$-effect. The main results of the present work are summarized in $\S 7$.

\section{THE HALL-MHD SYSTEM}

The dynamics of ideal and incompressible fully ionized plasmas in a rotating frame, is described by the induction equation (modified by the addition of the Hall current) and the Navier-Stokes equation,

$$
\begin{aligned}
\frac{\partial \boldsymbol{B}}{\partial t}= & \nabla \times[(\boldsymbol{U}-\lambda \nabla \times \boldsymbol{B}) \times \boldsymbol{B}] \\
\frac{\partial \boldsymbol{U}}{\partial t}= & -(\boldsymbol{U} \cdot \nabla) \boldsymbol{U}-2 \boldsymbol{\Omega} \times \boldsymbol{U}+(\nabla \times \boldsymbol{B}) \times \boldsymbol{B}- \\
& -\nabla\left(P-|\boldsymbol{\Omega} \times \boldsymbol{r}|^{2}\right)
\end{aligned}
$$

with the constraints

$$
\nabla \cdot \boldsymbol{U}=\nabla \cdot \boldsymbol{B}=0
$$

These equations are known as the Hall-MHD equations. The magnetic field is expressed in velocity units, i.e. $\boldsymbol{B}=\mathcal{B}[$ Gauss $](4 \pi \rho)^{-1 / 2}$, where $\rho$ is the constant mass density. The quantity $P$ is the gas pressure divided by the constant mass density. We define a dimensionless number $\epsilon$ to measure the relative strength of the Hall effect,

$$
\epsilon=\frac{\lambda}{L_{0}}
$$

where $L_{0}$ is the characteristic length scale of the system. If we choose $U_{0}=U_{A}$ as the characteristic velocity, $\lambda$ reduces to the ion skin depth. Note that equation (2) is valid for a fully ionized plasma.

\section{WAVES IN HALL-MHD}

We will study waves in the ideal and incompressible Hall-MHD system in a rotating frame. To linearize the Hall-MHD equations around a static and uniform magnetic field $\boldsymbol{B}_{0}$, we write

$$
\begin{aligned}
\boldsymbol{B} & =\boldsymbol{B}_{0}+\boldsymbol{b} \\
\boldsymbol{U} & =\boldsymbol{u},
\end{aligned}
$$

where $\boldsymbol{u}, \boldsymbol{b} \sim \exp (i \boldsymbol{k} \cdot \boldsymbol{r}-i \omega t)$. These substitutions convert (3) and (4) into the closed set :

$$
\begin{aligned}
-\omega \boldsymbol{b} & =\boldsymbol{k} \times\left[(\boldsymbol{u}-i \lambda \boldsymbol{k} \times \boldsymbol{b}) \times \boldsymbol{B}_{0}\right] \\
-\omega \boldsymbol{u} & =2 i \boldsymbol{\Omega} \times \boldsymbol{u}+(\boldsymbol{k} \times \boldsymbol{b}) \times \boldsymbol{B}_{0}-\boldsymbol{k} P_{t o t}
\end{aligned}
$$

where the total effective pressure $P_{t o t}=P-|\boldsymbol{\Omega} \times \boldsymbol{r}|^{2}$.

The elimination of $P_{t o t}$ in incompressible flows is arranged by projecting onto the plane perpendicular to $\boldsymbol{k}$. One finally obtains

$$
\begin{aligned}
-\omega \boldsymbol{b} & =\left(\boldsymbol{k} \cdot \boldsymbol{B}_{0}\right)(\boldsymbol{u}-i \lambda \boldsymbol{k} \times \boldsymbol{b}) \\
-\omega \boldsymbol{u} & =\mathbb{P}\left[2 i \boldsymbol{\Omega} \times \boldsymbol{u}+(\boldsymbol{k} \times \boldsymbol{b}) \times \boldsymbol{B}_{0}\right],
\end{aligned}
$$

where $\mathbb{P}_{i j}=\delta_{i j}-k_{i} k_{j} k^{-2}$ is the projector operator.

Without loss of generality we choose $\boldsymbol{k}$ in the $z$ direction, i.e. $\boldsymbol{k}=k \hat{z}$, while $\boldsymbol{\Omega}$ and $\boldsymbol{B}_{0}$ can be oriented arbitrarily. Equations (11) and (12), then, reduce to

$$
\begin{gathered}
\left(\omega \mathbb{I}+i \lambda k^{2} B_{z} \mathbb{A}\right) \boldsymbol{b}=-k B_{z} \mathbb{I} \boldsymbol{u} \\
\left(\omega \mathbb{I}-2 i \Omega_{z} \mathbb{A}\right) \boldsymbol{u}=-k B_{z} \mathbb{I} \boldsymbol{b} .
\end{gathered}
$$

Here,

$$
\mathbb{I}=\left(\begin{array}{lll}
1 & 0 & 0 \\
0 & 1 & 0 \\
0 & 0 & 0
\end{array}\right),
$$

and

$$
\mathbb{A}=\left(\begin{array}{rrr}
0 & 1 & 0 \\
-1 & 0 & 0 \\
0 & 0 & 0
\end{array}\right)
$$

Note that only the $z$-components of $\boldsymbol{\Omega}$ and $\boldsymbol{B}_{0}$ are relevant (i.e. the components in the direction of the vector $\boldsymbol{k}$ ). The orthonormal base for the antisymmetric operator $\mathbb{A}$ is given by

$$
| \pm\rangle=\frac{1}{\sqrt{2}}\left(\begin{array}{r}
1 \\
\pm i \\
0
\end{array}\right)
$$


and satisfies $\mathbb{A}| \pm\rangle= \pm i| \pm\rangle$.

The dispersion relation can be obtained from Equations (13) and (14),

$$
\omega^{2}-k^{2}\left(B_{z}^{2}+2 \lambda \Omega_{z} B_{z}\right)+\sigma \omega\left(2 \Omega_{z}-\lambda k^{2} B_{z}\right)=0,
$$

where $\sigma \equiv \pm 1$ and the eigenvectors can be written as $|\sigma\rangle$.

This dispersion relationship is quite general and includes several well known waves of a magnetized plasma in the corresponding asymptotic limits. When both $\Omega_{z}=0$ (no rotation) and $\lambda=0$ (negligible Hall current, i.e. the MHD limit) we obtain $\omega^{2}-k^{2} B_{z}^{2}=0$ which corresponds to Alfvén waves. In the incompressible limit, the shear and the compressional waves are degenerate and indistinguishable. When $\lambda=0$, equation (18) reduces to $\omega^{2}+2 \sigma \omega \Omega_{z}-k^{2} B_{z}^{2}=0$, and we obtain the inertial waves first described in the absence of an external magnetic field $B_{z}$ by Moffatt (1970a,b, 1972). Finally, when $\Omega_{z}=0$ we obtain two branches of circularly polarized waves. The right hand polarized branch, corresponds to whistlers, with a frequency growing like $\omega \simeq k^{2}$ at large wavenumbers. These waves are the high $\mathrm{k}$ limit of the compressional branch. The left handed branch is the standard shear wave whose frequency approaches the ion-cyclotron frequency asymptotically.

Figure 1 shows these asymptotic cases in detail. The general dispersion relationship given in equation (18), can be cast in dimensionless units using $L_{0}$ and $B_{z}=U_{A}$ as typical longitude and velocity,

$$
\omega^{2}-k^{2}(1+p \epsilon)+\sigma \omega\left(p-\epsilon k^{2}\right)=0,
$$

where

$$
p=\frac{2 \Omega_{z} L_{0}}{B_{z}},
$$

can be interpreted as the inverse of the Rossby number given in Eq. (1). The MHD limit in Figure 1 corresponds to a small neighborhood around the origin, where there is only a transition between inertial waves and Alfvén waves.

Figure 2 shows the phase speed as a function of wavenumber for the two positive branches given by equation (19). There are also two other identical branches with negative frequencies (not shown). These waves behave approximately as Alfvén waves only in the wavenumber regions where they are nondispersive, i.e. when the curves become horizontal.

\section{MHD WAVES AND THE DYNAMO EFFECT}

Before embarking on the Hall-MHD case, it is instructive to recall some previous results relating to the dynamo process induced by MHD waves ( readily obtained from our expressions). In the limit $\boldsymbol{\Omega} \rightarrow 0$ and $\lambda \rightarrow 0$, Alfvén waves are known to quench the $\alpha$-effect (Gruzinov \& Diamond 1994). The MHD $\alpha$ coefficient (Pouquet, Frisch, \& Léorat 1976; Blackman \& Field 1999) is given by

$$
\alpha=\frac{\tau}{3}(-\langle\boldsymbol{u} \cdot \nabla \times \boldsymbol{u}\rangle+\langle\boldsymbol{b} \cdot \nabla \times \boldsymbol{b}\rangle),
$$

where $\boldsymbol{u}$ and $\boldsymbol{b}$ are respectively small scale velocity and magnetic fields, unaffected by the presence of a large scale magnetic field (see details in Blackman \& Field (1999)), and the coefficient $\tau$ is a typical correlation time for the turbulent small scale motions. A pure Alfvenic state satisfies $\boldsymbol{u}= \pm \boldsymbol{b}$ and therefore $\alpha=0$. This is to be expected, since all nonlinear terms cancel exactly for Alvenic states, and therefore no transportation coefficients can arise in that case. On the other hand, when $\Omega \neq 0$ the Coriolis force is expected to inject helicity to the fluid, and therefore a net $\alpha$-effect arises (Moffatt 1978). A detailed discussion can also be found in Moffatt (1970a,b, 1972)

\section{HALL-MHD NORMAL MODES AND THE $\alpha$-EFFECT}

The first studies on the impact of Hall currents on dynamo action (Helmis 1968; Helmis 1971) were carried out using mean field theory and the first-order smoothing approximation (Krause \& Rädler 1980). Helmis obtained decreasing dynamo action as the strength of the Hall terms increased. Recently, the relevance of the Hall effect on dynamo activity was confirmed experimentally by Ding et al. (2004).

In Mininni, Gómez, \& Mahajan (2002a) it was shown that the expression for the $\alpha$-effect in the presence of the Hall effect is modified according to

$$
\begin{aligned}
\alpha= & \frac{\tau}{3}\left(-\left\langle\boldsymbol{u}^{e} \cdot \nabla \times \boldsymbol{u}^{e}\right\rangle+\langle\boldsymbol{b} \cdot \nabla \times \boldsymbol{b}\rangle-\right. \\
& \left.-\lambda\left\langle\boldsymbol{b} \cdot \nabla \times \nabla \times \boldsymbol{u}^{e}\right\rangle\right),
\end{aligned}
$$

where $\boldsymbol{u}^{e} \equiv \boldsymbol{u}-\lambda \nabla \times \boldsymbol{b}$ is the small scale electron flow velocity. This general expression differs from the MHD result (equation (21)) in two ways: it replaces the kinetic helicity by the helicity of the electron flow, and it contains an extra term due to the Hall current 
in the microscale. A nontrivial consequence of the latter is that, while the original $\alpha$ coefficient of Pouquet, Frisch, \& Léorat (1976) is zero for a pure Alfvénic state $\boldsymbol{u}=\sigma \boldsymbol{b}$ (Gruzinov \& Diamond 1994), the one corresponding to equation (22) is not. In Mininni, Gómez, \& Mahajan (2003b) the impact of the Hall effect in helical turbulent dynamos was studied in direct numerical simulations. As mentioned in the previous section, the required helicity is naturally introduced in a fluid in a rotating body by the Coriolis force.

Two questions arise. The first one is better posed and answered for a non-rotating plasma. We just showed that the $\alpha$ derived in MHD goes to zero for the pure Alfvénic state, which is an eigen-state of MHD. We also claimed that for the same pure eigenstate of MHD, the $\alpha$ derived in Hall MHD does not vanish. Surely that is an unwarranted mixing of different worlds. What we should, instead, calculate is the value of $\alpha$ derived in Hall MHD for the corresponding normal modes of Hall MHD. This task is performed at the end of this section.

The second question concerns rotation and the Coriolis force which is known to inject helicity at large scales (see equation [1] and Figure 1). At small scales (large $k$ ), however, the Coriolis force is not considered to be relevant (motions are expected to be essentially non-helical) posing a serious restriction on the generation of magnetic fields in astrophysics. Can we find a source of helicity acting at small scales, and will this source have much to do with rotation?

We show that the Hall effect naturally introduces helicity at small scales, which is precisely the region where this effect is stronger (in agreement with Wardle (1999) and also Balbus \& Terquem (2001)). However, no net $\alpha$-effect is generated by these microscale motions, unless there is also a net rotation of the system; a combination of rotation and Hall effect is needed for dynamo action.

As is shown in equation (17), the general solutions of the linearized Hall-MHD equations in a rotating frame are right-handed or left-handed polarized waves. To investigate the effect of Hall currents at small scales, we first concentrate on a non-rotating plasma, $\Omega_{z}=0$. For large $\mathrm{k}$, the dispersion relationship (18) reduces to

$$
\omega^{2}-k^{2} B_{z}^{2}-\sigma \lambda \omega k^{2} B_{z}=0,
$$

which coupled to Eq.(11), yields

$$
\boldsymbol{b}=-\frac{k B_{z}}{\omega} \boldsymbol{u}_{e}
$$

Inserting Eq. (24) into equation (22), and invoking that the electron vorticity is $\nabla \times \boldsymbol{u}_{e}=\sigma k \boldsymbol{u}_{e}$, we obtain

$\alpha=-\frac{\tau}{3}\left(\frac{\omega^{2}-k^{2} B_{z}^{2}-\sigma \lambda \omega k^{2} B_{z}}{\omega^{2}}\right)\left\langle\boldsymbol{u}^{e} \cdot \nabla \times \boldsymbol{u}^{e}\right\rangle$

which, according to the dispersion relation Eq. (23), corresponds to $\alpha=0$. This result of a zero $\alpha$-effect for Hall MHD normal modes is an expected and natural extension of the similar result obtained for Alfvenic states in MHD. However, we must bear in mind that these results are derived for non-rotating systems.

\section{CORIOLIS FORCE AND THE $\alpha$-EFFECT}

In rotating objects, the closure calculation leading to either equation (22) (in the MHD limit) or equation (21) needs to be revised, since the Coriolis force (see equation (4)) was not included in those calculations. The starting point is the so-called "reduced smoothing approximation" (RSA) proposed by Blackman \& Field (1999) (see also Mininni, Gómez, \& Mahajan (2003a) for a derivation which includes the Hall effect). Following RSA, we decompose the magnetic and velocity fields as

$$
\begin{gathered}
\boldsymbol{B}=\overline{\boldsymbol{B}}+\boldsymbol{b}+\boldsymbol{b}^{0} \\
\boldsymbol{U}=\overline{\boldsymbol{U}}+\boldsymbol{u}+\boldsymbol{u}^{0}
\end{gathered}
$$

where the overbar denotes spatially or statistically averaged large-scale perturbations. The small scale fields $\boldsymbol{b}^{0}, \boldsymbol{u}^{0}$ are solutions of Eqs. (3)-(4) in the absence of large scale fields, and $\boldsymbol{b}, \boldsymbol{u}$ are anisotropic corrections to the small scale fields, caused by the presence of $\overline{\boldsymbol{B}}, \overline{\boldsymbol{U}}$. The net effect of small scale fluctuations on the large scale dynamics, is given by an electromotive force

$$
\mathcal{E}=\left\langle\boldsymbol{u}^{0, e} \times \boldsymbol{b}+\boldsymbol{u}^{e} \times \boldsymbol{b}^{0}\right\rangle
$$

acting on equation (4), where $\boldsymbol{u}^{e}=\boldsymbol{u}-\lambda \nabla \times \boldsymbol{b}$ and $\boldsymbol{u}^{0, e}=\boldsymbol{u}^{0}-\lambda \nabla \times \boldsymbol{b}^{0}$.

From equations (3)-(4) for the evolution of small scale fields (assuming that $\overline{\boldsymbol{U}} \approx 0$ in an appropriate frame of reference, and under the reduced smoothing approximation (Blackman \& Field 1999)), we obtain

$$
\begin{gathered}
\partial_{t} \boldsymbol{b} \simeq(\overline{\boldsymbol{B}} \cdot \nabla) \boldsymbol{u}^{0, e}, \\
{\left[\partial_{t}+2 \mathbb{I} \cdot \boldsymbol{\Omega} \times\right] \boldsymbol{u} \simeq(\overline{\boldsymbol{B}} \cdot \nabla) \boldsymbol{b}^{0} .}
\end{gathered}
$$


The time derivative indicated in equations (29)-(30) is usually approximated by $\partial_{t} \approx 1 / \tau$, where $\tau$ is a correlation time for the microscale motions. The operator on the left-hand side of equation (30) becomes,

$$
\mathbb{T}^{-1}=\frac{1}{\tau}+2 \mathbb{I} \cdot \mathbf{\Omega} \times
$$

whose inverse is

$$
\mathbb{T}=\frac{\tau}{1+\left(2 \Omega_{z} \tau\right)^{2}}\left(\mathbb{I}+2 \Omega_{z} \tau \mathbb{A}\right)
$$

Replacing equations (29)-(30) on equation (28) and using equation (32), we obtain

$$
\begin{aligned}
\mathcal{E}= & \frac{\tau \sigma k B_{z}}{\omega^{2}}\left(-\omega^{2}+\frac{k^{2} B_{z}^{2}}{1+\left(2 \Omega_{z} \tau\right)^{2}}+\right. \\
& \left.+\lambda \sigma k^{2} B_{z} \omega\right)\left\langle\left|\boldsymbol{u}^{0, e}\right|^{2}\right\rangle .
\end{aligned}
$$

The expression for the electromotive force given in equation (33) corresponds to an anisotropic tensor

$$
\begin{aligned}
\alpha_{i j}=- & \frac{\sigma k \tau}{\omega^{2}}\left\langle\left|\boldsymbol{u}^{0, e}\right|^{2}\right\rangle\left[2 \Omega_{z}\left(\lambda k^{2} B_{z}-\sigma \omega\right)+\right. \\
& \left.+k^{2} B_{z}^{2}\left(\frac{\left(2 \Omega_{z} \tau\right)^{2}}{1+\left(2 \Omega_{z} \tau\right)^{2}}\right)\right] \frac{k_{i} k_{j}}{k^{2}}
\end{aligned}
$$

which for small scale fluctuations given by a pure mode of wavenumber $\boldsymbol{k}$, produce an electromotive force $\mathcal{E}$ parallel to $\boldsymbol{k}$, regardless of the orientation of $\overline{\boldsymbol{B}}$ and $\Omega$. It seems reasonable to assume that the correlation time $\tau$ is much smaller than the rotation period. Therefore, in the asymptotic limit $\Omega \tau \ll 1$,

$$
\alpha_{i j}=-\frac{2 \Omega_{z} \tau k}{\omega}\left\langle\left|\boldsymbol{u}^{0, e}\right|^{2}\right\rangle\left[\frac{\lambda \sigma k^{2} B_{z}}{\omega}-1\right] \frac{k_{i} k_{j}}{k^{2}} .
$$

Note that when a rotation field is present, $\alpha_{i j}$ is in general non-zero. The rotation field therefore provides a source of energy that is responsible for the net $\alpha$ effect. Equation (35) confirms that for non-rotating objects, the net alpha effect generated by a background of small scale normal modes is exactly zero. Notwithstanding, sources of kinetic helicity other than rotation have been considered (Mininni, Gómez, \& Mahajan 2003a,b), to assess their efficiency in driving large scale dynamos.

For large wavenumbers, the dispersion relationship given by equation (18) has two limits: one is the so called compressional/whistler branch $\omega \approx \pm \lambda k^{2} B_{z}$, while the other is the shear/cyclotron branch with $\omega \approx \pm B_{z} / \lambda$. For the former, the $\alpha$-effect is asymptotically small. For the shear/cyclotron branch, however, $\alpha_{i j} \propto k^{3}$ at large wavenumbers implying strong dynamo action. Therefore, once the rotation breaks the mirror symmetry, the shear/ion-cyclotron modes ( which failed to produce dynamo action without rotation) are, indeed, able to provide a net $\alpha$-effect at microscopic scales. We believe that this is a very important result for turbulent dynamo theories. In Figure 3 we show the trace of the tensor $\alpha_{i j}$ as a function of $k$, for different values of $p$ and $\epsilon$. Note that in the MHD case $\alpha_{i i}$ drops to zero at small scales (large $k$ ), while in the Hall-MHD case it does not.

\section{DISCUSSION}

We have shown that the Hall effect in conjunction with fluid rotation can generate helicity at small scales (i.e. produce small scale helical motions) leading to a net $\alpha$-effect through the agency of the shear/ioncyclotron normal mode of the plasma. This finding can be of considerable importance to the existence of large scale dynamo action in a variety of astrophysical objects. Our results are quite consistent with previous results obtained in the study of instabilities in accretion disks. Wardle (1999), Balbus \& Terquem (2001), and Sano \& Stone (2002) showed that the magneto-rotational instability can be either enhanced or quenched by the Hall effect depending on the orientation of $\boldsymbol{\Omega}$ and $\boldsymbol{B}_{0}$, which is just a manifestation of the handedness introduced by the Hall effect. In a future work the detailed investigation of this mode of dynamo action will be carried out through direct numerical simulations.

The authors are grateful to Dr. A. Pouquet for very fruitful and enlightening comments. Research of SMM was supported by US DOE contract DEFG03-96ER-54366 Research of DOG and PDM has been funded by grant X209/01 from the University of Buenos Aires. PDM is a fellow of CONICET, and DOG is a member of the Carrera del Investigador Científico of CONICET.

\section{REFERENCES}

Balbus, S.A. \& Terquem, C. 2001, ApJ, 552, 235

Blackman, E.G. \& Field, G.B. 1999, ApJ, 521, 597

Brandenburg, A. 2001, ApJ, 550, 824 
Chen, Q., Chen, S., \& Eyink, G. 2003, Phys. Fluids, 15, 361

Ding, W.X. and 8 co-authors 2004, Phys. Rev. Lett., 93, 045002

Geppert, U, \& Rheinhardt, M. 2002, Astron. \& Astrophys., 392, 1015

Gómez, D. \& Mininni, P. 2004, Phys. A, in press.

Gruzinov, A. \& Diamond, P.H. 1994, Phys. Rev. Lett., 72,1651

Helmis, G. 1968, Mber. Dtsch. Akad. Wiss. Berlin, 10, 280

Helmis, G. 1971, Beitr. Plasma Physik, 11417

Ji, H. 1999, Phys. Rev. Lett., 83, 3198

Krause, F. \& Rädler, K.-H., 1980, Mean-Field Magnetohydrodynamics and Dynamo Theory, (GDR: Pergamon Press)

Meneguzzi, M., Frisch, U., \& Pouquet, A. 1981, Phys. Rev. Lett., 47, 1060

Mininni, P.D., Gómez, D.O., \& Mahajan, S.M. 2002, ApJ, 567, L81

Mininni, P.D., Gómez, D.O., \& Mahajan, S.M. 2003, ApJ, 584, 1120

Mininni, P.D., Gómez, D.O., \& Mahajan, S.M. 2003, ApJ, 587, 472

Moffatt, H.K. 1970, J. Fluid. Mech., 41, 435

Moffatt, H.K. 1970, J. Fluid. Mech., 44, 705

Moffatt, H.K. 1972, J. Fluid. Mech., 53, 385

Moffatt, H.K. 1978, Magnetic field generation in electrically conducting fluids (Cambridge: Cambridge University Press)

Pouquet, A., Frisch, U., \& Léorat, J. 1976, J. Fluid Mech., 77, 321

Rädler, K.-H. 1969, Monatsber. Dt. Akad. Wiss., 11, 194

Sano, T. \& Stone, J.M. 2002, ApJ, 570, 314.

Spruit, H.C. 2002, Astron. \& Astrophys. 381, 932

Urpin, V. 2002, Phys. Rev. E65, 026301
Wardle, M. 1999, MNRAS, 307, 849 


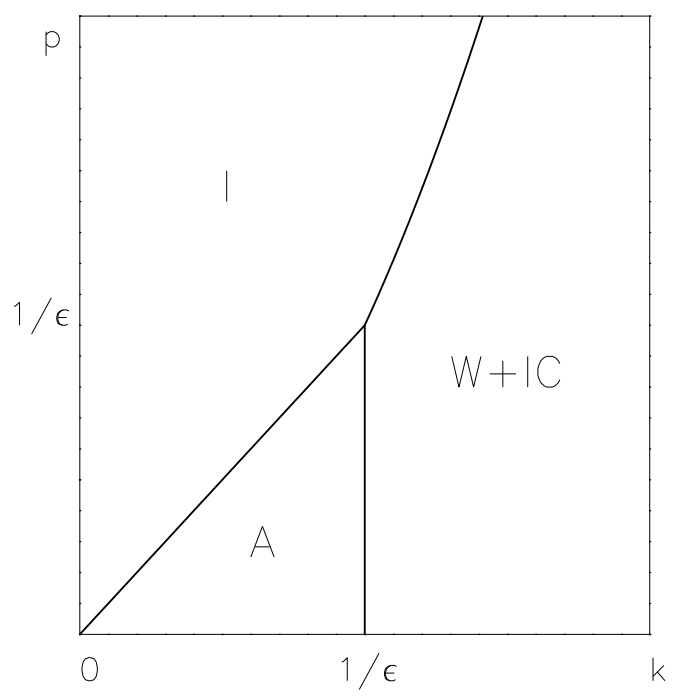

Fig. 1.- Regions dominated by: whistlers (W) and ion-cyclotron (IC) waves, inertial (I), and Alfvén (A) waves. The horizontal axis is the wavenumber in units of $1 / L_{0}$. The vertical axis corresponds to $p=$ $2 \Omega_{z} L_{0} / B_{z}$.
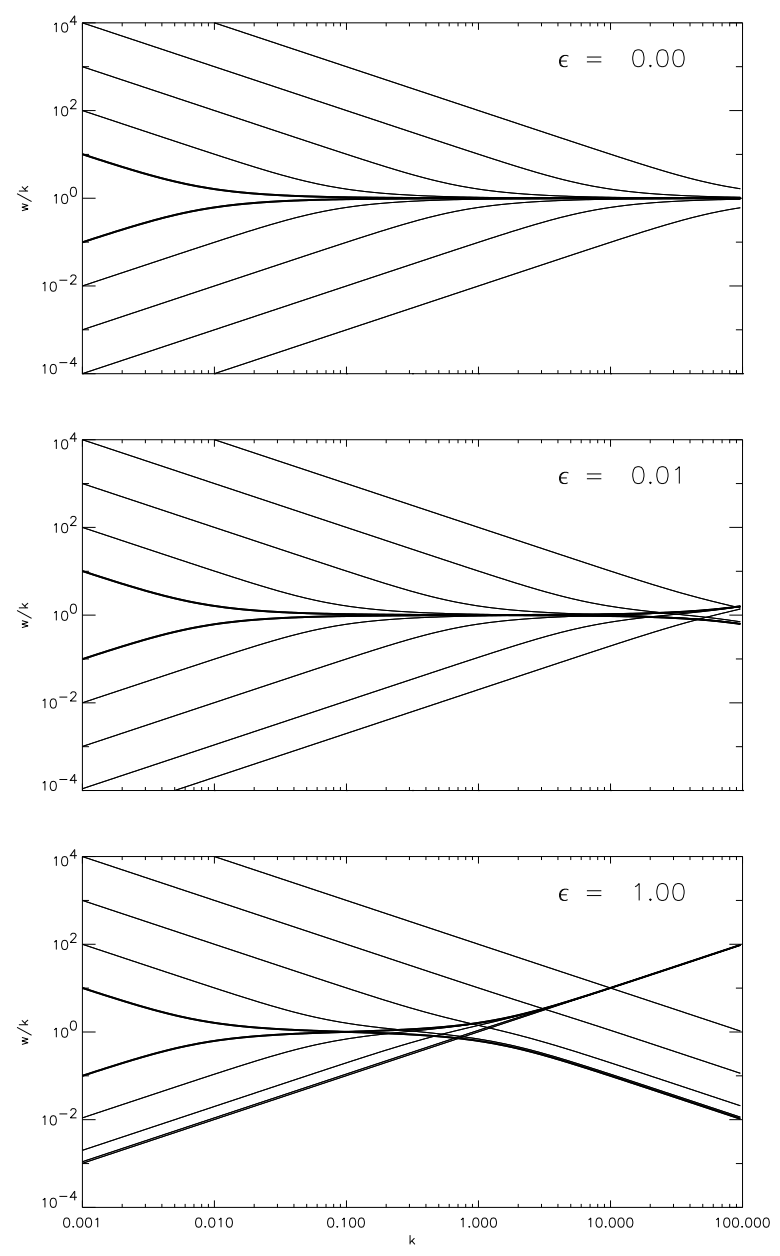

Fig. 2.- Phase speed vs. wavenumber for the normal modes arising from the normalized dispersion relationship given in equation (19). Each frame corresponds to a different value of $\epsilon$, while different traces correspond to $p=0.01$ (thick) and $p=0.1,1,10,100$. 

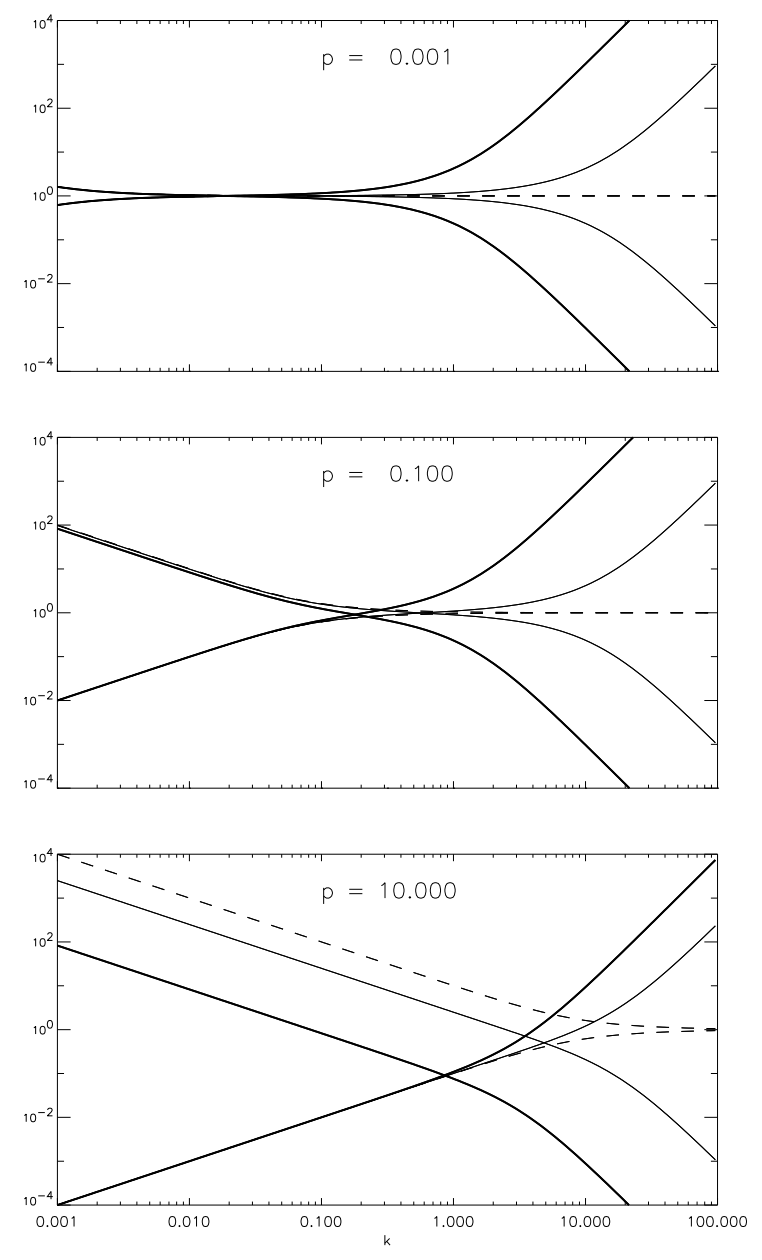

Fig. 3.- The trace of $\alpha_{i j}$ vs. k in arbitrary units (see equation (35) for the two positive branches of the dispersion relationship. Each frame corresponds to a different value of the dimensionless rotation speed $p$ (indicated). The dotted curves correspond to $\epsilon=0$, the thin full curves to $\epsilon=0.1$, and the thick full curves to $\epsilon=1$. 